\title{
International Journal of
}

\section{Intelligent Systems and Applications}

\section{Kol.4}

No. 8 Jul. 2012
IJISA Kol.4

Http:// www.mecs-press .org Vol. 4 No.8 July 2012

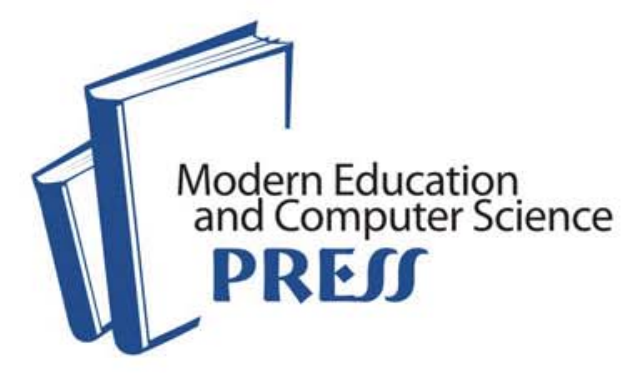


International Journal of Intelligent Systems and Applications (IJISA)

ISSN Print: 2074-904X, ISSN Online: 2074-9058

Volume 4, Number 8, July 2012

\section{Contents}

\section{REGULAR PAPERS}

Phone Duration Modeling of Affective Speech Using Support Vector Regression

Alexandros Lazaridis, Iosif Mporas, Todor Ganchev

On Some Topological Properties of Pessimistic Multigranular Rough Sets

B.K.Tripathy,M. Nagaraju

Design of Type-2 Fuzzy Controller based on LQR Mapped Fusion Function

Abhishek Kumar,Sudeep Sharma,R. Mitra

Design and Implementation of Face Recognition System in Matlab Using the Features of Lips

Sasikumar Gurumurthy,B.K.Tripathy

Internet Traffic Classification for Educational Institutions Using Machine Learning

Jaspreet Kaur,Sunil Agrawal,B.S.Sohi

Research on Key Technology of New Concept Tyre Building Production Line

Menglong Cao, Guangbo Wang, Lina Sun

Supervised Online Adaptive Control of Inverted Pendulum System Using ADALINE Artificial Neural Network

with Varying System Parameters and External Disturbance

Sudeep Sharma, Vijay Kumar,Raj Kumar

Two Approaches Based on Genetic Algorithm to Generate Short Iris Codes 